\title{
Automated Tortuosity Analysis of Nerve Fibers in Corneal Confocal Microscopy
}

\author{
Yitian Zhao, Jiong Zhang, Ella Pereira, Yalin Zheng, Pan Su, Jianyang Xie, Yifan Zhao, Yonggang Shi, \\ Hong Qi, Jiang Liu, Yonghuai Liu
}

\begin{abstract}
Precise characterization and analysis of corneal nerve fiber tortuosity are of great importance in facilitating examination and diagnosis of many eye-related diseases. In this paper we propose a fully automated method for image-level tortuosity estimation, comprising image enhancement, exponential curvature estimation, and tortuosity level classification. The image enhancement component is based on an extended Retinex model, which not only corrects imbalanced illumination and improves image contrast in an image, but also models noise explicitly to aid removal of imaging noise. Afterwards, we take advantage of exponential curvature estimation in the 3D space of positions and orientations to directly measure curvature based on the enhanced images, rather than relying on the explicit segmentation and skeletonization steps in a conventional pipeline usually with accumulated preprocessing errors. The proposed method has been applied over two corneal nerve microscopy datasets for the estimation of a tortuosity level for each image. The experimental results show that it performs better than several selected state-of-the-art methods. Furthermore, we have performed manual gradings at tortuosity level of four hundred and three corneal nerve microscopic images, and this dataset has been released for public access to facilitate other researchers in the community in carrying out further research on the same and related topics.
\end{abstract}

Index Terms-Corneal nerve, tortuosity, enhancement, segmentation, curvature.

Copyright (c) 2019 IEEE. Personal use of this material is permitted. However, permission to use this material for any other purposes must be obtained from the IEEE by sending a request to pubspermissions@ieee.org.

This work was supported by Zhejiang Provincial Natural Science Foundation of China (LZ19F010001), the Key Research and Development Program of Zhejiang Province (2020C030360), National Science Foundation Program of China (61906181), China Postdoctoral Science Foundation (2019M652156), Ningbo "2025 S\&T Megaprojects" (2019B10033, 2019B10061) and Ningbo Natural Science Foundation (2018A610055).

Y. Zhao, P. Su, and J. Xie are with Cixi Institute of Biomedical Engineering, Ningbo Institute of Materials Technology and Engineering, Chinese Academy of Sciences, Ningbo 315201, China; J. Zhang and Y. Shi are with Keck School of Medicine, University of Southern California, Los Angeles 90033, US; E. Pereira and Y. Liu are with Department of Computer Science, Edge Hill University, Ormskirk L39 4QP, UK; Y. Zheng is with Department of Eye and Vision Science, University of Liverpool, Liverpool L69 3BX, UK; Yifan Zhao is with the School of Aerospace, Transport and Manufacturing, Cranfield University, Bedford MK43 OAL, UK; H. Qi is with Department of Ophthalmology, Peking University Third Hospital, Beijing 100191, China; J. Liu is with Department of Computer Science and Engineering, Southern University of Science and Technology, Shenzhen 518055, China.

Corresponding author: Jiong Zhang (jiong.zhang@loni.usc.edu)

\section{INTRODUCTION}

Existing clinical research [1], [2] suggests that the morphological changes observable in numerous anatomical curvilinear structures - e.g., retinal blood vessels, coronary blood vessel, carotid or corneal nerve fibers - are closely related to the presence of many diseases. For example, several studies [1][6] have confirmed that some quantitative properties of the corneal nerves such as nerve fiber branching, density, length, and tortuosity are linked to eye and systemic diseases. Corneal nerve damage and repair from surgical interventions may be reflected in nerve branching, while fungal keratitis patients usually exhibit lower nerve density [7]. Tortuosity is one of the most significant biomarkers reflecting variations in corneal nerve fibers, as larger tortuosity implies process of nerve degeneration and subsequent regeneration, leading to active neural growth [2]. In addition, ophthalmologists often use the tortuosity of corneal nerve fibers as an important clinical parameter to assess hypertensive retinopathy [8] and diabetic neuropathy [9]-[12], respectively.

Corneal Confocal Microscopy (CCM) imaging is a common procedure to visualize and inspect corneal nerves - in particular the subbasal nerve plexus - due to its non-invasive nature of acquisition [9]. In several studies [3], [9], [13], corneal nerve fibre tortuosity has been categorized into between three and five levels, or simply as normal/abnormal. However, these assessments are usually subjective, and can lead to substantial inter-observer and intra-observer variations [3]. Fig. 1 illustrates three examples of CCM images with increasing tortuosity. Clinicians have suggested that any measure of tortuosity should be invariant to translation, rotation and scaling [2]. An accurate measurement of tortuosity should be able to extract the key tortuosity characteristics - e.g., amplitude and number of inflection points (frequency) - and to overcome time constraints and inaccurate measurements.

The conventional approaches for automatic tortuosity measurement start from a segmentation step of curvilinear structures, followed by a skeletonization to obtain a centerline map. Afterwards, junction points such as bifurcations and crossings are detected and then used to break the centerline map into individual centerline segments. For each segment, a curve fitting and parameterization step is applied to achieve the best approximation to the original curvilinear structures. The local curvatures of each centerline segment are finally estimated for the purpose of generating different tortuosity metrics.

To our best knowledge, the tortuosity of curvilinear struc- 


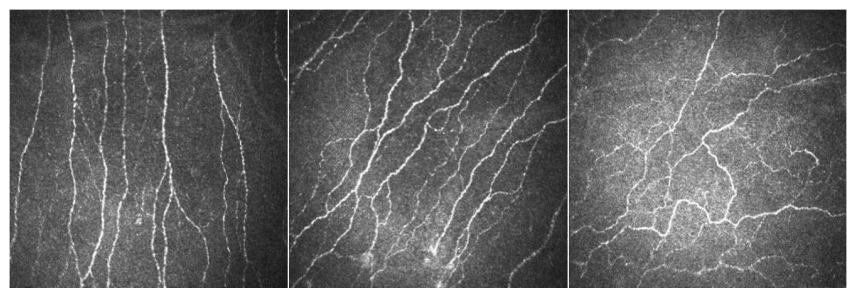

Fig. 1. Three examples of corneal nerve images with different tortuosity levels. From left to right: levels 1 to 3.

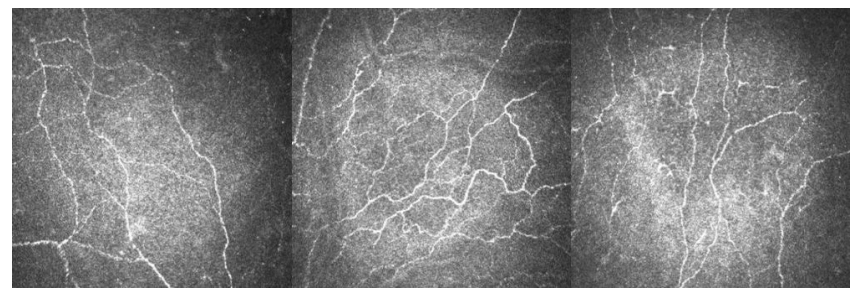

Fig. 2. Examples of CCMs with poor contrast, inhomogeneous backgrounds and presence of noise during image acquisition.

tures has no universal accepted definition or standard description. It has different characteristics for different anatomical structures [8], [9], [14], [15]. Most tortuosity measurements are historically rooted in metrics designed for quantifying the curvature of curvilinear structures such as retinal vessels [8], cerebral blood vessels [16], and corneal nerves [17]. Bracher [18] suggests that tortuosity may be obtained by computing the ratio (ARC) of the distance traveled (arcs length) and the straight-line distance between contour endpoints (chord length). However, this approach ignores inflection points, and tends to assign the same value to potentially very different shapes. Bullitt et al. [16] further multiply the ARC by the number of inflection points along the retinal vessels, while Grisan et al. [8] sum the ARC computed from each vessel between inflection points, and then multiply by the number of inflection points. Semdby et al. [19] measure tortuosity through a sum of angles metric, which evaluates the angles between consecutive trios of points along the space curve represented by the vessel skeleton. This measure is effective in detecting high-frequency, low-amplitude coils or sine waves. Goh et al. [20] measure tortuosity by computing the change of angles calculated at reasonable discrete steps along the blood vessel, and direction change along the path of the vessels, respectively. Bribiesca [21] proposes a measure of tortuosity for retinal blood vessels based on slope chain code. This method places constant-length straight-line segments along the curve, and computes the slope changes between contiguous segments scaled to a continuous range.

Hart et al. [22] use total curvature and total squared curvature along the vessel centerlines to distinguish the retinal blood vessels as simply tortuous or non-tortuous. 18 tortuosity measures are calculated from a corneal nerve dataset by Annunziata et al. [3], [13]. The researchers employ the multinomial logistic ordinal regressor to identify the best combination of tortuosity measures, and the nerves are graded into four levels of tortuosity. Scarpa et al. [9] adapt the algorithm [8] for the analysis of corneal nerve tortuosity by further partitioning each nerve into several turn curves, which are located between two consecutive twists. The twists are denoted by changes in curvature sign between the contour endpoints.

\section{A. Challenges}

Most of the aforementioned tortuosity measures may lead to disagreement, in particular that an image may be perceptually classified by an ophthalmologist as highly tortuous even though it contains only one or two nerves with many little twists [3]. In addition, manual analysis of nerve fibers by ophthalmologists is a tedious task and prone to human errors, while existing commercial software analysis still largely relies on manual refinement. On the other hand, the accuracy of a computational tortuosity measure is highly dependent on the quality of pre-processing: errors can be propagated and accumulated due to poor imaging quality, and imperfect curvilinear structure segmentation or tracing. The imaging quality of CCM remains an issue of concern for the development of automated fiber detection and tortuosity estimation [17]. As a consequence, a fully automated and reliable assessment of the tortuosity level of curvilinear structures has long been deemed desirable to overcome time constraints and avoid human error.

Automated corneal nerve fiber analysis methods, such as fiber segmentation or tortuosity estimation, are particularly challenging due to poor contrast, imaging noise, and/or imbalanced intensity, as shown in Fig. 2. Intensity inhomogeneity and poor contrast, and/or speckle noise are often inherited from the medical image acquisition process [23]. These obstacles pose significant challenges to subsequent fiber detection and tortuosity estimation tasks. To this end, it is also crucial to produce high-quality enhancements of the captured images so as to reveal significant details obscured inside.

\section{B. Contributions}

Being well acquainted with the above challenges, we propose a novel method that seamlessly integrates two distinct technical components, image enhancement and tortuosity estimation, with the underlying ideas that each of them will address one of the aforementioned challenges. More specifically, this paper makes three contributions:

- We propose a novel algorithm to improve the quality of CCM images, by modeling noise explicitly in the classic Retinex model, so that different components - illumination, reflectance, and noise - can be estimated simultaneously. To this end, an objective function is constructed by minimizing their difference, gradient, and magnitude and optimized using the powerful alternating direction method of multipliers.

- To better describe the tortuosity of curvilinear structures, we introduce an advanced exponential curvature estimation method, with a view to avoid the unstable factors in the conventional approach. Exponential curvature estimation has the great advantage of measuring the curvature values directly from the enhanced images, rather than relying on the complex pre-processing steps in the conventional pipeline.

- The proposed framework has been validated quantitatively using two corneal nerve tortuosity datasets and one 
retinal blood vessel tortuosity dataset. In addition, we have performed manual annotations of nerve fibers in these corneal nerve datasets, and constructed a new corneal nerve tortuosity dataset. All these datasets have been released for public access.

\section{Corneal Confocal Microscopy Enhancement}

In this work, we propose an image enhancement algorithm to improve the quality of CCM imagery and reduce the noise by considering a noise term explicitly in the classic Retinex model [24]. Using this model, we make a first attempt to predict the noise map by applying the Retinex model to the medical images, while simultaneously estimating a structureenhanced reflectance map.

Throughout this paper, the following notations are used: the low case letter in italics denotes a scalar, the upper case letter denotes a matrix, the lower case letter in bold face denotes a vector, the vectorization of a matrix $X$ is represented as $\mathbf{x}$, the operations on matrices or vectors such as multiplication ', less than or equal to $\leq$, and division / is performed elementwise, unless stated otherwise, and superscript $T$ denotes the transpose of a vector or matrix.

\section{A. Preliminaries}

The classic Retinex model assumes that a given image $S$ can be decomposed into two components, the reflectance $R$ and the illumination $L$ :

$$
S=L \cdot R, \quad \text { s.t. } \quad S \leq L .
$$

where $S, L$, and $R$ are matrices, whose element values are in the unit interval $[0,1]$. The goal is to estimate the reflectance $R$ and the illumination $L$ from $S$. Once $R$ is estimated reliably, it can be regarded as the desired enhanced image $S$ for the more faithful representation of the reflectance of the object of interest and for the purpose of subsequent analysis. Most existing Retinex methods [25]-[28] utilize logarithmic operations to linearize the model and reduce computational complexity, resulting in $\log (S)=\log (L)+\log (R)$.

To estimate $L$ and $R$, the conventional methods [29], [30] usually build and optimize the following objective function:

$$
\begin{gathered}
\underset{R, L}{\operatorname{argmin}}\|R \cdot L-S\|_{2}+\alpha\|\nabla L\|+\beta\|\nabla R\|_{2}, \\
\text { s.t. } \quad R \leq 1 \text { and } S \leq L .
\end{gathered}
$$

In this objective function, $\alpha$ and $\beta$ are used to control the tradeoff between the fidelity term and the gradient term. $\|\cdot\|_{2}$ and $\|\cdot\|$ are the $l_{2}$ and $l_{1}$ norms, respectively. However, a direct estimation of the reflectance may lead to excessive smoothing, and remove semantically significant edge details. Moreover, the gradient variation in the log-transformed reflectance is represented as: $\nabla(\log (R))=(1 / R) \cdot \nabla R$, and so is heavily influenced by $1 / R$ when $R$ is very small. $1 / R$ becomes highly unstable if $R$ contains intensive noise (which is a common phenomenon in the CCM images), and it often amplifies the latent intensive noise due to a lack of constraint on the reflectance [31], [32].

\section{B. Noise-constrained Retinex model}

In order to avoid the above-mentioned issues, in this work we do not apply logarithmic transformation to the Retinex model, instead we introduce a noise map $N$ into the conventional Retinex model directly with a view to enhance images corrupted by intensive noise. Our model is thus defined as:

$$
S=L \cdot R+N .
$$

The proposed objective function that simultaneously estimates the reflectance $R$, the illumination $L$ and the noise $N$ from the given image $S$ can then be defined as:

$$
\underset{R, L, N}{\operatorname{argmin}}\|R \cdot L+N-S\|_{2}+\alpha\|\nabla L\|+\beta\|\nabla R-G\|_{2}+\sigma\|N\|_{2},
$$

where $R \leq 1, S \leq L$, and we use the $l_{2}$ norm to constrain the overall intensity of noise $N$. The fidelity term $\|R \cdot L+N-S\|_{2}$ is the squared difference between the $S$ and the recomposed one $R \cdot L+N$. The second term $\nabla L$ denotes the regularization term, which is employed to preserve the edges and details and compress noise. The term $\|\nabla R-G\|_{2}$ minimizes the difference between the gradient of the reflectance $R$ and $G$, where $G$ is the adjusted gradient of $S$, so that the structure of the edges of the reflectance is smooth and continuous. In this work, $G$ is the amplified version of the gradient of the input image with a factor $K$, such that: $G=K \cdot \nabla S$, where $K=1+\lambda e^{-|\nabla S| / \delta}$. This amplification operation ensures less adjustment in regions with higher gradient magnitudes, while regions with lower gradient magnitudes are strongly enhanced [32]. As a result, the adjusted gradient $G$ tends to reach an even magnitude. $\lambda$ and $\delta$ control the degree of the amplification and the amplification rate of different gradients.

It is clear that Eqn. (4) is an ill-posed inverse image decomposition problem to recover three variables $R, L$ and $N$ from only one known variable $S$. In this case, an approach giving a convergence guarantee for non-convex optimization problems will be required. To this end, we employ the Alternating Direction Method of Multipliers (ADMM) [33] to optimize this objective function. By introducing an auxiliary variable $B$ [34], we can rewrite the objective function in Eqn. (4) as:

$$
\begin{gathered}
\underset{R, L, B, N}{\operatorname{argmin}}\|R \cdot L+N-S\|_{2}+\alpha\|B\|+\beta\|\nabla R-G\|_{2}+\sigma\|N\|_{2}, \\
\text { s.t. } \quad B=\nabla L .
\end{gathered}
$$

\section{Solutions to the optimization problem}

In this subsection, we provide the solutions to the subproblems in Eqn. (5) in the $k^{t h}$ iteration.

- $L$ sub-problem: To optimize the above objective function in Eqn. (5), we introduce a Lagrange multiplier $Z$ to remove the equality constraint, so as to iteratively update each variable, while regarding the others estimated in the previous iteration as constants. By neglecting the unrelated terms of $L$, the objective function in Eqn. (5) can be rewritten as:

$$
\underset{L}{\operatorname{argmin}}\|R \cdot L+N-S\|_{2}+\Phi\left(Z^{k}, \nabla L-B^{k}\right),
$$




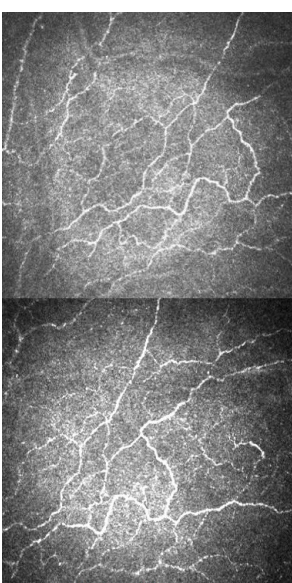

Original image

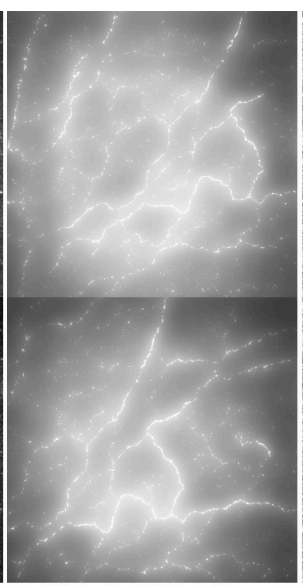

Illumination map

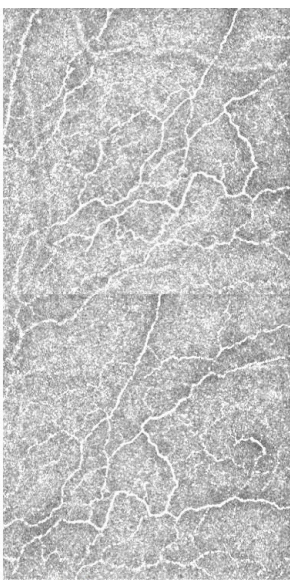

Reflectance map

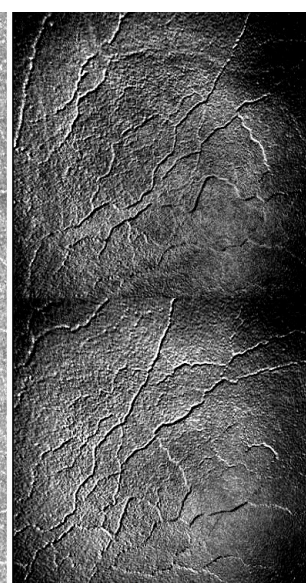

Noise map

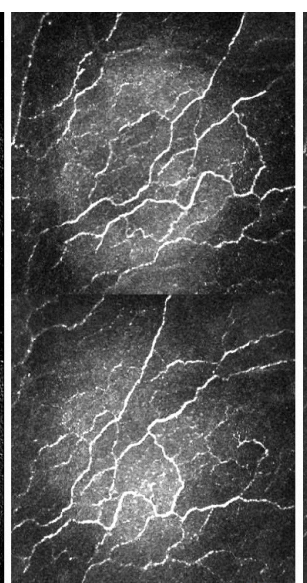

no gamma correction

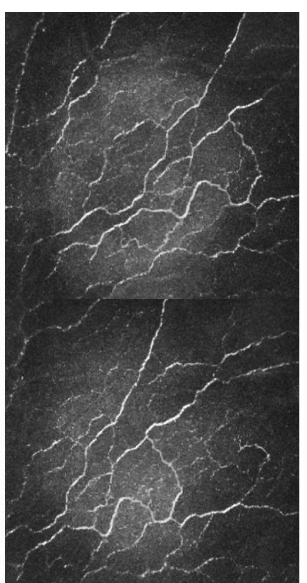

Enhanced image

Fig. 3. Examples of image decomposition and corresponding enhancement results. From left to right: original CCM images, illumination maps, reflectance maps, noise maps and enhanced images.

where $\Phi(\mathrm{X}, \mathrm{Y})=\langle\mathrm{X}, \mathrm{Y}\rangle+\frac{\mu}{2}\|Y\|_{2}$ as suggested by [32], and $\langle\cdot, \cdot\rangle$ represents the matrix inner product, and the solution to $L$ is then derived as:

$$
\begin{aligned}
& \mathbf{l}^{k+1}=\left(2 f\left(\mathbf{r}^{k+1}\right)+\mu f(\mathrm{D})\right)^{-1} \\
& \quad\left(2 \mathbf{r}^{k+1}\left(\mathbf{s}-\mathbf{n}^{k+1}\right)+\mu \mathrm{D}^{T}\left(\mathbf{b}^{k}-\frac{\mathbf{z}^{k}}{\mu}\right)\right),
\end{aligned}
$$

where $\mu$ is the penalty scalar, $\mathrm{D}$ is the discrete gradient operator; and $\mu^{k+1}=1.5 \cdot \mu^{k}$; and $f(X)=X^{T} X$.

- $R$ sub-problem: Similarly, by neglecting the unrelated terms of $R$, the objective function in Eqn. (5) is rewritten as:

$$
\underset{R}{\operatorname{argmin}}\|R \cdot L+N-S\|_{2}+\beta\|\nabla R-G\|_{2} .
$$

We further replace the first term as a classical least squares problem:

$$
\underset{R}{\operatorname{argmin}}\|\mathbf{r} \cdot \mathbf{l}+\mathbf{n}-\mathbf{s}\|_{2}+\beta\|\nabla R-G\|_{2} .
$$

By differentiating the above equation with respect to $R$ and setting the derivative to 0 , we have

$$
\begin{aligned}
& 2\left(\mathbf{l}^{k}\right)^{T}\left(\mathbf{l}^{k} \mathbf{r}+\mathbf{n}^{k}-\mathbf{s}\right)+2 \beta \mathrm{D}^{T}(\mathrm{Dr}-\mathbf{g})=0, \text { leading to } \\
& \left(f\left(\mathbf{l}^{k}\right)+\beta f(\mathrm{D})\right) \mathbf{r}=\mathbf{l}^{k}\left(\mathbf{s}-\mathbf{n}^{k}\right)+\beta \mathrm{D}^{T} \mathbf{g} \text { and thus } \\
& \mathbf{r}^{k+1}=\left(f\left(\mathbf{l}^{k}\right)+\beta f(\mathrm{D})\right)^{-1}\left(\mathbf{l}^{k}\left(\mathbf{s}-\mathbf{n}^{k}\right)+\beta \mathrm{D}^{T} \mathbf{g}\right) .
\end{aligned}
$$

- $N$ sub-problem: By neglecting the unrelated terms of $N$, the objective function in Eqn. (5) can be rewritten as:

$$
\underset{N}{\operatorname{argmin}}\|R \cdot L+N-S\|_{2}+\sigma\|N\|_{2} .
$$

The closed form solution to this quadratic problem is:

$$
N^{k+1}=\left(S-R^{k+1} \cdot L^{k+1}\right) /(1+\sigma) .
$$

- $B$ sub-problem: By neglecting the unrelated terms of $B$, the objective function in Eqn. (5) can be rewritten as:

$$
\underset{B}{\operatorname{argmin}} \alpha\|B\|+\Phi\left(Z^{k}, \nabla L^{k+1}-B\right) .
$$

A shrinkage operation is adopted to update $B^{k+1}$, giving the solution to Eqn. (13) as:

$$
B^{k+1}=\operatorname{shrink}_{\epsilon}\left(\nabla L^{k+1}+\frac{Z^{k}}{\mu^{k}}\right),
$$

where $\operatorname{shrink}_{\epsilon}(\cdot)=\operatorname{sign}(\cdot) \max (|\cdot|-\epsilon, 0)$ and $\epsilon=\frac{\alpha}{\mu^{k}}$. This solution can be expanded as:

$$
B^{k+1}=\operatorname{sign}\left(\nabla L^{k+1}+\frac{Z^{k}}{\mu^{k}}\right) \cdot \max \left(\left|\nabla L^{k+1}+\frac{Z^{k}}{\mu^{k}}\right|-\frac{\alpha}{\mu^{k}}, 0\right),
$$

The auxiliary matrix $Z$ can be finally updated as $Z^{k+1}=$ $Z^{k}+\mu^{k}\left(\nabla L^{k+1}-B^{k+1}\right)$.

\section{Implementation details}

We set initial values of $L, N, Z, B, k$ and $\mu$ as $L^{0}=S$, $N^{0}=Z^{0}=B^{0}=0, k=0$, and $\mu^{0}=1$. The whole process is repeated until the difference between $R^{k}$ and $R^{k+1}$ is smaller than 0.001. After having estimated the illumination and the reflectance maps, illumination adjustment is performed to enhance the detail of the input image, by employing a Gamma correction of $L$ with $L^{\prime}=L^{\frac{1}{\gamma}}$, where $\gamma$ is empirically set as 1.8. Many Retinex-based image enhancement methods are accompanied with gamma correction such as those proposed in [35]-[37]. They use the gamma correction to compress the dynamic range of the illumination, and reduce the rapid changes in actual lighting, so as to improve the visibility of the input image. In this work, we employed the gamma correction in order to prevent over-enhancement and under-enhancement for a good balance between foreground and background. The enhanced image $\hat{S}$ produced by the proposed method is finally estimated as $\hat{S}=L^{\prime} \cdot R$.

Fig. 3 illustrates the decomposed illumination, reflectance, and noise maps, as well as the proposed enhancement results without and with gamma correction applied. It can be observed that the enhanced results (right column of Fig. 3) maintain the overall structure of the illumination, suppress the majority of the noisy granular texture and present better visual quality by revealing greater local details about the nerve fibers.

\section{Curvature-Based Tortuosity Estimation}

As curvature-based tortuosity biomarkers are valuable features to quantify the development of disease complications, accurate curvature computation is highly needed to describe 
the tortuosity changes of enhanced corneal nerve fibers. However, curvature measurement in conventional pipelines requires complicated segmentation and skeletonization steps which may cause inaccurate estimation due to accumulated preprocessing errors. To precisely describe nerve fiber geometric variations, we set up a tortuosity measurement framework based on the direct estimation of curvatures over the enhanced images to avoid the unstable factors in the conventional approach. The theoretical basics for setting up the exponential curvature estimation and the details for obtaining the curvature-based nerve fiber tortuosity biomarkers will be explained in the following subsections.

\section{A. Curvilinear data representation using orientation scores}

Exponential curvature is defined in the domain of positions and orientations $\mathbb{R}^{2} \rtimes S^{1}$, which is identified by the group of planar translations $\mathbf{x}=(x, y) \in R^{2}$ and rotations $\theta \in[0,2 \pi]$ with group element $g=(\mathbf{x}, \theta)$. The group product in this domain is defined as

$g g^{\prime}=(\mathbf{x}, \theta)\left(\mathbf{x}^{\prime}, \theta^{\prime}\right)=\left(\mathbf{x}+\mathbf{R}_{\theta} \cdot \mathbf{x}^{\prime}, \theta+\theta^{\prime}\right)$, for all $g, g^{\prime} \in \mathbb{R}^{2} \rtimes S^{1}$, where $\mathbf{R}_{\theta}=\left(\begin{array}{cc}\cos \theta & -\sin \theta \\ \sin \theta & \cos \theta\end{array}\right)$ is a counter-clockwise rotation over angle $\theta$. The semi-direct product " $\rtimes$ " indicates that translations are accompanied with rotations in $S E(2)$ since a rotation $\mathbf{R}_{\theta}$ pops up in the translation part. The domain of translations and rotations $\mathbb{R}^{2} \rtimes S^{1}$ provides the geometric basics for analyzing curvilinear structures like corneal nerve fibers in a new space. In $\mathbb{R}^{2} \rtimes S^{1}$, the tangent vector $X_{e}$ of a curve $\gamma: \mathbb{R} \rightarrow S E(2)$ at the origin $\mathbf{o}=(0,0,0)^{T}$ is spanned by the unit basis $e=\left\{\mathbf{e}_{x}, \mathbf{e}_{y}, \mathbf{e}_{\theta}\right\}$ of positions and orientations. After this curve has been translated by $\mathbf{x}$ and rotated by $\theta$, the tangent vector $X_{g}$ to this new curve can be transformed in a left-invariant way with $g=(\mathbf{x}, \theta)$, so that both $X_{e}$ and $X_{g}$ have the same components with a new Euclidean invariant basis [38] for the effective operation of the original image:

$$
\left\{\left.\mathbf{e}_{\xi}\right|_{g},\left.\mathbf{e}_{\eta}\right|_{g},\left.\mathbf{e}_{\theta}\right|_{g}\right\}=\left\{\cos \theta \mathbf{e}_{x}+\sin \theta \mathbf{e}_{y}, \cos \theta \mathbf{e}_{y}-\sin \theta \mathbf{e}_{x}, \mathbf{e}_{\theta}\right\} .
$$

To analyze the curvilinear structures in the domain $\mathbb{R}^{2} \rtimes S^{1}$, the enhanced 2D image obtained from the previous section is first mapped to a 3D space of positions and orientations via a wavelet-type transform, creating orientation scores (OS) [38], [39] (Fig. 4(a)-(b)). The transform is achieved through convolution, with an anisotropic kernel $\psi \in \mathbb{L}_{2}\left(\mathbb{R}^{2}\right)$ :

$$
U_{f}(\mathbf{x}, \theta)=\int_{\mathbb{R}^{2}} \overline{\psi\left(\mathbf{R}_{\theta}^{-1}(\mathbf{y}-\mathbf{x})\right)} f(\mathbf{y}) d \mathbf{y},
$$

where $U_{f}$ represents an orientation score, constructed from image $f$ via the oriented cake wavelet transform, and depends on two variables $(\mathbf{x}, \theta)$. It actually represents the orientation responses of each spatial location $(x, y)$ at different angles $\theta$. The OS of the curved geometry in $\mathbb{R}^{2} \rtimes S^{1}$ can lift and separate crossed curvilinear structures and provide a novel representation of data for calculating their curvatures in the $3 \mathrm{D}$ space, rather than in the normal 2D image space.

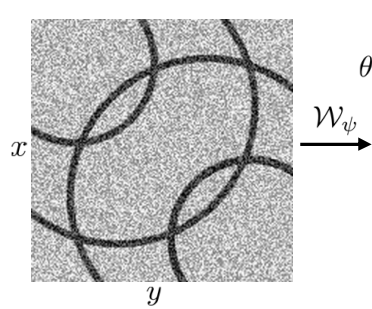

(a)Synthetic image

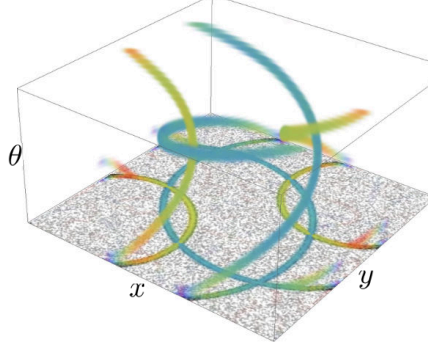

(c)Curvature in $\mathbb{R}^{2} \rtimes S^{1}$

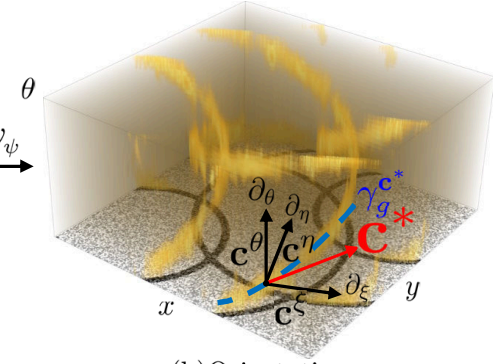

(b)Orientation score

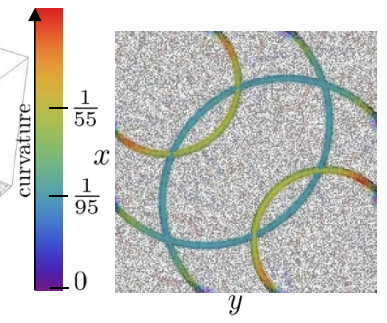

(d)Projected curvature in $\mathbb{R}^{2}$
Fig. 4. Validation of the exponential curvature estimation in $\mathbb{R}^{2} \rtimes S^{1}$ on (a) a typical synthetic image with $\mathrm{SNR}=1$; (b) shows the lifted data representation in orientation scores; (c) gives the color-coded curvature map, obtained using the exponential curve fit for the estimation of optimal tangent vector $\mathbf{c}^{*}$; (d) shows the projected curvature map in the $2 \mathrm{D}$ image domain.

\section{B. Local curvature $\kappa$ from the best fit exponential curves}

An exponential curve is defined as a curve $\gamma_{\mathbf{c}}$ in $\mathbb{R}^{2} \rtimes S^{1}$ with constant tangent vector components $\mathbf{c}=\left(c^{\xi}, c^{\eta}, c^{\theta}\right)^{T}$ expressed in the Euclidean-invariant basis $\left\{\mathbf{e}_{\xi}, \mathbf{e}_{\eta}, \mathbf{e}_{\theta}\right\}$ : i.e.,

$$
\dot{\gamma}_{\mathbf{c}}(t)=\left.c^{\xi} \mathbf{e}_{\xi}\right|_{\gamma_{\mathbf{c}}(t)}+\left.c^{\eta} \mathbf{e}_{\eta}\right|_{\gamma_{\mathbf{c}}(t)}+\left.c^{\theta} \mathbf{e}_{\theta}\right|_{\gamma_{\mathbf{c}}(t)},
$$

where $t \in \mathbb{R}$ is an arbitrary parameter for defining the arc length in the domain of $\mathbb{R}^{2} \rtimes S^{1}$. In orientation scores, exponential curves are similar to straight lines with respect to the curved geometry in $\mathbb{R}^{2} \rtimes S^{1}$. Their spatial projections directly define the curvature at each pixel, see Fig. 4 (b). Therefore, for a tangent vector $\mathbf{c}$ of the exponential curve, we can directly deduce the curvature of its spatially projected curves $\mathbb{P}_{\mathbb{R}^{2}} \gamma_{\mathbf{c}}$ from their tangent vector components via

$$
\kappa(\mathbf{x}, \theta)=\frac{c^{\theta} \operatorname{sign}\left(c^{\xi}\right)}{\sqrt{\left|c^{\xi}\right|^{2}+\left|c^{\eta}\right|^{2}}},
$$

i.e., the rate of angular change with respect to the spatial movement along the curve, as shown in Fig. 4(b).

The orientation scores provide rich angular information for each pixel in a 2D image. However, the local orientations in the score domain are not always perfectly aligned with local structure, as only a limited number of orientations is set in the transformation. Since we aim to estimate local curvatures by considering the corresponding angular changes, we need to ensure the best alignment between the oriented kernels and real data. As such, the exponential curve fit is employed to achieve the best alignment with locally oriented structures. This can provide more accurate local curvature estimation for corneal nerve fibers. The optimal tangent vector $\mathbf{c}^{*}$ (shown in red color in Fig. 4 (b)) of the best fit exponential curve is used 

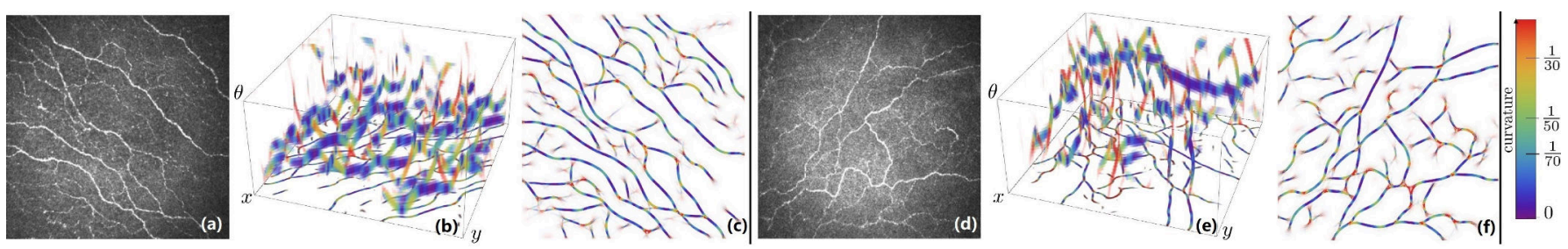

Fig. 5. Typical examples of the exponential curvature estimation applied to corneal nerve fiber images. (a) and (d) are original images; (b) and (e) give color-coded curvature maps in the lifted orientation score space; (c) and (f) show the projected curvature map in the 2D image domain.

to compute the local curvature $\kappa$. It can be obtained through the following minimization problem:

$$
\mathbf{c}^{*}(g)=\underset{\mathbf{c} \in \mathbb{R}^{3},\|\mathbf{c}\|_{\mu}=1}{\operatorname{argmin}}\left\{\left\|\left.\frac{d}{d t} \nabla U_{f}\left(\gamma_{g}^{\mathbf{c}}(t)\right)\right|_{t=0}\right\|_{\mu}^{2}\right\},
$$

with $\|\mathbf{c}\|_{\mu}^{2}=\mu^{2}\left|c^{\xi}\right|^{2}+\mu^{2}\left|c^{\eta}\right|^{2}+\left|c^{\theta}\right|^{2}=\left\|M_{\mu} \mathbf{c}\right\|^{2}$. Here we have $M_{\mu}=\operatorname{diag}(\mu, \mu, 1)$ with a dimension 1/length for $\mu$ [40], [41]. This is introduced to make sure that the distances in spatial and orientation dimensions in $\mathbb{R}^{2} \rtimes S^{1}$ are comparable and is empirically determined in practice. In this work, the optimal tangent vector $\mathbf{c}^{*}$ is found via eigensystem analysis of the Gaussian Hessian. We best fit the exponential curves to real corneal nerve fiber data via second-order Hessian features in orientation scores. Hence, the numerical method for solving $\mathbf{c}^{*}$ can be considered as obtaining the eigenvectors of the orientation score-based symmetrized and $\mu$ normalized Hessian matrix $\mathcal{H}_{\mu} U_{f}=M_{\mu^{-1}}\left(\mathcal{H} U_{f}\right)^{T} M_{\mu^{-2}}\left(\mathcal{H} U_{f}\right) M_{\mu^{-1}}$, with

$$
\mathcal{H} U_{f}=\left(\begin{array}{ccc}
\partial_{\xi}^{2} U_{f} & \partial_{\xi} \partial_{\eta} U_{f} & \partial_{\theta} \partial_{\xi} U_{f} \\
\partial_{\xi} \partial_{\eta} U_{f} & \partial_{\eta}^{2} U_{f} & \partial_{\theta} \partial_{\eta} U_{f} \\
\partial_{\xi} \partial_{\theta} U_{f} & \partial_{\eta} \partial_{\theta} U_{f} & \partial_{\theta}^{2} U_{f}
\end{array}\right)
$$

where it is computed via rotation-invariant derivative frames $\left\{\partial_{\xi}, \partial_{\eta}, \partial_{\theta}\right\}:=\left\{\cos \theta \partial_{x}+\sin \theta \partial_{y}, \cos \theta \partial_{y}-\sin \theta \partial_{x}, \partial_{\theta}\right\}$, and where we use the short hand notation $\left\{\partial_{x}, \partial_{y}\right\}$ for the basis instead of the conventional $\left\{\mathbf{e}_{x}, \mathbf{e}_{y}\right\}$. Hence, the final curvature measurement $\kappa_{\mathbf{c}^{*}}(\mathbf{x}, \theta)$ is obtained via the vector components of the optimal tangent vector $\mathbf{c}^{*}$. Fig. 4 shows an example of using the exponential curvature estimation on a synthetic image with curvilinear structures. Visual comparison shows an excellent agreement between the estimated curvature and the real curvature of the synthetic curves.

\section{Curvature-based tortuosity biomarker}

We take advantage of the proposed vessel enhancement technique to provide high-quality curvilinear structure maps for more accurate curvature estimation, rather than using the original images directly. In Fig. 5, we show two typical examples of curvature estimation on corneal nerve fiber images. The results show that the curvature maps capture local changes of curvilinear structures well. Our method is in contrast with an existing one [40], which estimates the curvature measure based on the 3D orientation confidence, obtained via the Laplacian computed in the cross-sectional plane orthogonal to the structures in orientation scores. The orientation confidence is estimated in an iterative way, and the curvature values are also updated and weighted accordingly

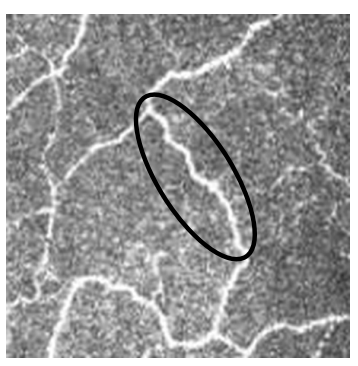

(a)Original image

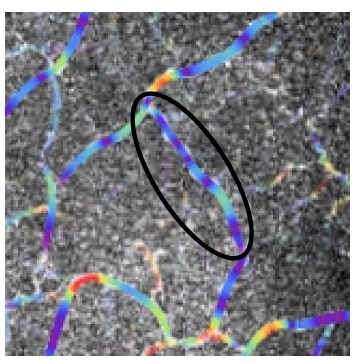

(c)Curvature of 20 iterations (d)Curvature of averaging

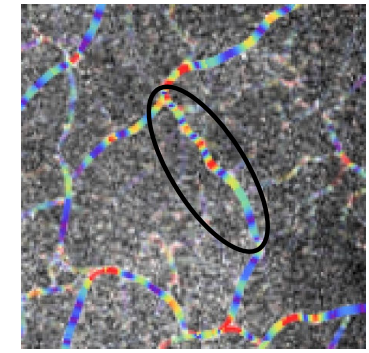

(b)Curvature of our setting

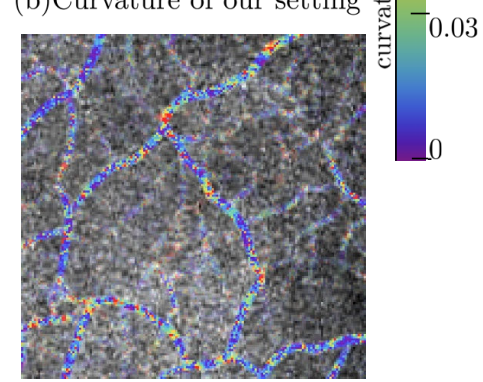

0.03
Fig. 6. Curvatures estimated using different methods or schemes. (a) The original image; (b) The projected curvature map based on our method with the maximum orientation response and no iterative refinement; (c) The curvature map of using the iterative method [40]; (d) The projected curvature map based on our method with the average orientation response.

using the newly estimated orientation confidence. One of our improvements is that we simplify this curvature calculation by directly computing curvature from the enhanced corneal nerve fiber maps rather than using the iterative refinement. This iterative refinement process may improve the precision of curvature measurement on smoothly elongated structures. However, it could also cause over-smoothing of the curvilinear structures when using a large number of iterations for refinement. This is particularly a case illustrated in Fig. 6(c), in which this iterative refinement process causes over-smoothing effect and thus produces an inaccurate estimation of curvatures over curved nerve fiber parts (the black frame for example). Fig. 6(b) shows the projected curvature map based on our approach.

The curvature-based global tortuosity biomarker in this work is defined as

$$
\begin{aligned}
\kappa_{\exp } & =\frac{1}{\mathcal{V}} \int_{-\infty}^{\infty}\left|\kappa_{\mathbf{c}^{*}}(\mathbf{x}, \Theta(\mathbf{x}))\right| \hat{S}(\mathbf{x}) \mathrm{d} \mathbf{x}, \text { with } \\
\Theta(\mathbf{x}) & =\underset{\theta_{i} \in \frac{\pi}{N o}\{1, \ldots, N o\}}{\arg \max }\left\{U\left(\mathbf{x}, \theta_{i}\right)\right\},
\end{aligned}
$$


where $U(\mathbf{x}, \theta)$ gives the orientation scores of the enhanced corneal nerve fibers $\hat{S}$ and where the total image summation $\mathcal{V}=\int_{-\infty}^{\infty} \hat{S}(\mathbf{x}) d \mathbf{x}$ is used for normalization. Thus, $\kappa_{\exp }$ represents the summation of the absolute curvatures that are weighted by our enhanced nerve fiber map $\hat{S}(\mathbf{x})$.

To emphasize local curvature changes, here we take the curvature value of the maximum orientation response at $\Theta(\mathbf{x})$ in the 3D space over all the orientations per position, instead of directly averaging the whole $3 \mathrm{D}$ volume for curvature measurement. This can help to better describe the local curvature changes rather than obscuring the local nerve fibre structure details. Two typical examples of the 2D projected curvature map using the maximum and average response schemes are shown in Fig. 6(b) and (d) respectively. We can observe that the averaged curvature results in fluctuated variations, while the maximum response provides a more stable and reliable curvature estimation without losing much structure details. For each image, we obtain the measure $\kappa_{\exp }$ as the exponential curvature-based biomarker for further tortuosity analysis.

\section{Tortuosity classification}

The final task is to assign a tortuosity grade to each image. In practice, each image contains variable numbers of blood vessels or nerve fibers with varying lengths, which lead to considerably different tortuosity characteristics [3]. Therefore, we use a weighted average operation to measure the tortuosity at image level. This takes into account the length $(l)$ of the $i^{\text {th }}$ curvilinear structure as the weight: $M=\frac{\sum_{i=1}^{n} l_{i} \times m_{i}}{\sum_{i=1}^{n} l_{i}}$, where $m$ is one of the tortuosity measures described in TABLE I, as well as our measure $\kappa_{\text {exp }}$. The total number of curvilinear segments within an image is $n$.

Finally, a total of 14 tortuosity measures are employed to form a feature vector per image. The classification of the tortuosity level is achieved based on the extracted imagelevel features by employing the linear Support Vector Machine (SVM). We employed the Matlab built-in linear SVM model with all the parameters set to default values. Given the modest size of the used datasets, we use a 10-fold cross-validation to make sure for SVM to produce reliable classification results. Note, we try to abide by Occam's razor, a principle that states that out of all the possible models that provide similar performance, the one that is the simplest should be selected as the final model.

\section{MATERIALS}

Here, we describe two datasets to be used in the next section for the evaluation of the proposed approach, including our newly-released corneal nerve fiber dataset (CCM-A) along with manual annotations of nerve fiber and tortuosity levels, and one existing publicly available dataset (CCM-B).

- CCM-A: This dataset was collected and created by the Peking University Third Hospital, China. 403 CCM images of corneal subbasal epithelium were acquired from 103 normal and pathological subjects using a Heidelberg Retina Tomograph equipped with a Rostock Cornea Module (HRTIII) microscope. The subject population included, 28 healthy subjects, 24 patients with diabetes, 28 subjects with dry eye
TABLE I

EXISTING TORTUOSITY MEASUREMENTS FOR CURVILINEAR STRUCTURES.

\begin{tabular}{c|lc}
\hline \hline No. & Tortuosity Measures & Notations \\
\hline 1 & Chord Length [18] & $L \chi$ \\
2 & Curve Length [18] & $L_{c}$ \\
3 & Arc Length over Chord Length Ratio [42] & $\tau$ \\
4 & Absolute Curvature [22] & $\kappa_{a}$ \\
5 & Squared Curvature [22] & $\kappa_{s}$ \\
6 & Absolute Curvature Weighted by Curve Length [43] & $\kappa_{a c}$ \\
7 & Tortuosity Density [8] & TD \\
8 & Tortuosity Coefficient [44] & TC \\
9 & Slope Chain Coding [45] & SCC \\
10 & Directional Change of a Line [46] & DCI \\
11 & Mean Direction Angle Change [47] & MAC \\
12 & Absolute Direction Angle Change [20] & AAC \\
13 & Inflection Count Metric [16] & ICM \\
\hline
\end{tabular}

disease, and 23 patients with both dry eye and diabetes. Each image has a resolution of $384 \times 384$ pixels covering a field of view of $400 \times 400 \mu \mathrm{m}^{2}$. The reference fiber centerlines were manually annotated by an ophthalmologist using the open source software ImageJ. These images were further categorized into four groups based on fiber tortuosity level. An image analysis expert and the clinical author (obs 1 and $o b s$ 2) each independently labeled the tortuosity level according to a previously published protocol [48], and the consensus between them was then used as ground truth $(G T)$, i.e., Level 1: the fibers appear almost straight (54 images); Level 2: the fibers appear moderately tortuous (212 images); Level 3: the fibers are quite tortuous; the amplitude of the changes in the fiber direction is quite severe (108 images); Level 4: the fibers appear very tortuous, presenting frequent changes in the fiber direction (29 images). We have made this dataset available online ${ }^{1}$.

- CCM-B: This is a publicly available database [9] constructed by University of Padova, Italy. The database is composed of $60 \mathrm{CCM}$ images, which were acquired from normal and pathologic subjects (diabetes, pseudoex foliation syndrome, and keratoconus), using an HRT-II microscope. An expert grouped all the images by the degree of tortuosity into three different classes - low, mid, and high. In addition, one of our clinical authors traced the centerlines of all the visible nerves, and this manual annotation has also been released for public access alongside CCM-A.

\section{EXPERIMENTAL RESULTS}

In order to validate the effectiveness and superiority of the proposed method, we evaluate separately its individual components: image enhancement, tortuosity grading and tortursity measurement in the following subsections.

\section{A. Evaluation on image enhancement}

Firstly, we analyze the effect of our noise-constrained Retinex pre-processing step in the proposed method. In practice, the $\lambda$ and $\delta$ are empirically set as 10 .

\footnotetext{
${ }^{1}$ http: //imed.nimte.ac.cn/
} 


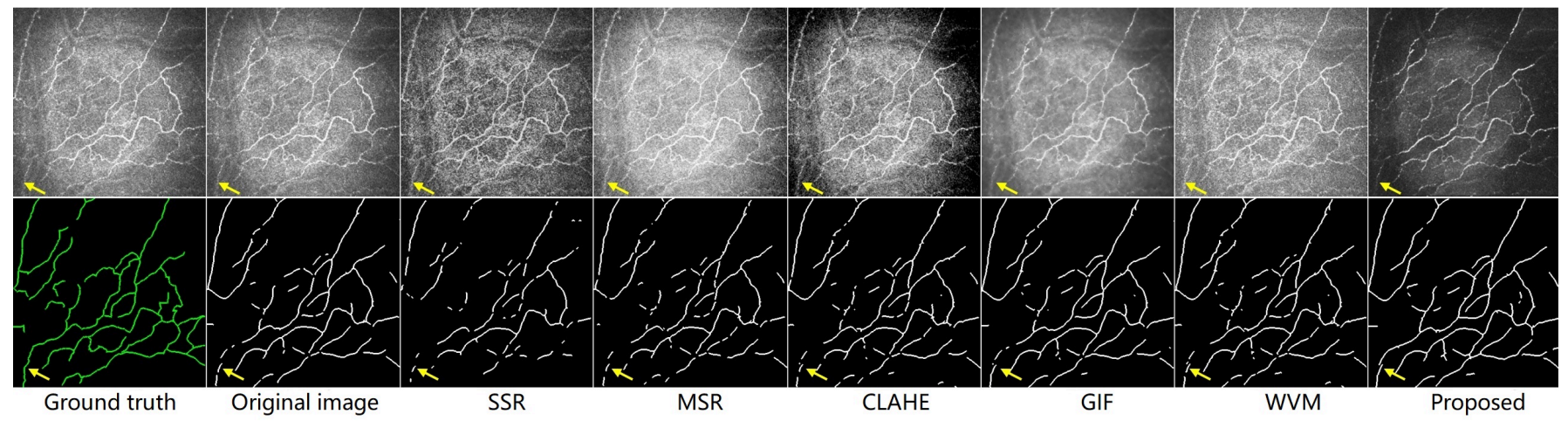

Fig. 7. An example of image enhancement result from CCM-A using different enhancement methods, and its guided nerve fiber tracing result using the IPACHR segmentation method [49].

1) Subjective comparisons: The top row of Fig. 7 presents the enhancement results produced by the proposed enhancement method, and other five state-of-the-art approaches: Single Scale Retinex (SSR) [50], Multi Scale Retinex (MSR) [51], Contrast-Limited Adaptive Histogram Equalization (CLAHE) [52], Guided Image Filter (GIF) [53], and Weighted Variational Model (WVM) [54]. SSR and MSR attempt to remove the effect of illumination on the given image in order to enhance its contrast. However, these two methods generate noticeable artifacts or noise in flat regions, as the continuous values of adjacent pixels are stretched apart. This is a side effect of the logarithmic transformation discussed in Section II-A.

CLAHE enhances the image uniformly, irrespective of whether a given region is in the foreground or background. SSR can easily over-enhance regions with relatively high intensities. MSR and GIF provide over-smoothed results, and do not preserve well fine structures such as smaller fibers. In contrast, the WVM model and the proposed method yielded more visually informative results.

2) Evaluation by using SNR: It is difficult to demonstrate conclusively the superiority of the proposed enhancement method purely by the above visual inspection. In this subsection, we compute the signal to noise ratio (SNR): $S N R=$ $10 \log _{10}\left(m_{n} / m_{b}\right) \mathrm{dB}$, where $m_{n}$ and $m_{b}$ are the mean pixel intensity of the nerves and the mean pixel intensity of the background respectively. As aforementioned, the nerve fibres have been manually traced, and we define the regions after a disk-shaped dilation operation on the manual traced fibres with a radius $(r)$ of 5 and 9 pixels, respectively, as the background. Fig. 8 illustrates an example of these background regions (green label) in an original and enhanced image.

The quantitative results of different enhancement approaches are shown in TABLE II. The proposed method has achieved the best performance - it exhibits a large advantage against the original images by an increase in SNR of about $4.60 \mathrm{~dB}$ and $6.70 \mathrm{~dB}$ when $r=5$ and $r=9$, respectively. Comparatively, our method is able to reduce inhomogeneities due to imbalanced intensity, and normalize the entire background to a similar level, so as to increase the contrast between the nerve fibers and their background, as expected.

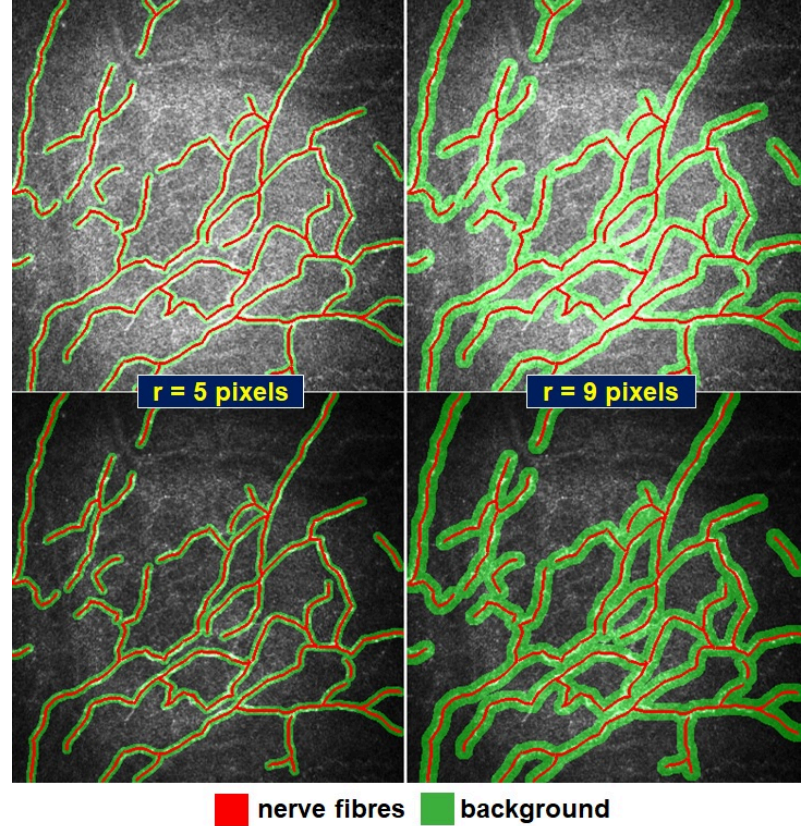

Fig. 8. An example to show the regions selected as background so as to compute the SNR. The background was determined by a dilation operation on the manual traced fibres (red color) with a radius of 5 and 9 pixels, respectively. Top row: an original image; Bottom tow: the enhanced image.

3) Image enhancement-guided fiber segmentation: In this section, we perform corneal nerve fiber segmentation of the enhanced images to confirm the relative benefits of the proposed method and the others.

We obtained two sets of corneal nerve fiber segmentation results both with and without the application of enhancement methods. Two different segmentation methods were selected to extract the corneal nerves from the original and enhanced images respectively: 1) a deep learning-based method; and 2) an infinite active contour-based segmentation method. Deep learning methods have demonstrated superior performance and better prospects for many medical image segmentation problems. In this work, we employed one of the most latest curvilinear structure segmentation network: CS-Net [55], for fully automatic segmentation of corneal nerves, with and without application of image enhancement methods. We trained 
TABLE II

THE RESULTS OF SNR OF THE ORIGINAL AND ENHANCED IMAGES PRODUCED BY DIFFERENT METHODS.

\begin{tabular}{l|cc}
\hline \hline & $\mathrm{r}=5$ & $\mathrm{r}=9$ \\
\hline Raw & $13.54 \pm 0.05 \mathrm{~dB}$ & $14.21 \pm 0.10 \mathrm{~dB}$ \\
\hline SSR & $15.23 \pm 0.04 \mathrm{~dB}$ & $14.96 \pm 0.09 \mathrm{~dB}$ \\
MSR & $12.98 \pm 0.11 \mathrm{~dB}$ & $13.24 \pm 0.10 \mathrm{~dB}$ \\
CLAHE & $14.37 \pm 0.09 \mathrm{~dB}$ & $15.17 \pm 0.11 \mathrm{~dB}$ \\
GIF & $16.98 \pm 0.07 \mathrm{~dB}$ & $14.55 \pm 0.06 \mathrm{~dB}$ \\
WVM & $16.56 \pm 0.09 \mathrm{~dB}$ & $18.43 \pm 0.08 \mathrm{~dB}$ \\
\hline Proposed & $\mathbf{1 8 . 1 4} \pm \mathbf{0 . 0 6} \mathrm{dB}$ & $\mathbf{2 0 . 9 1} \pm \mathbf{0 . 0 4} \mathrm{dB}$ \\
\hline
\end{tabular}

TABLE III

SEGMENTATION RESULTS OBTAINED USING DIFFERENT METHODS WITH DIFFERENT ENHANCEMENT METHODS APPLIED TO CCM-A.

\begin{tabular}{l|cc|cc}
\hline \hline \multirow{2}{*}{} & \multicolumn{2}{|c|}{ IPACHR } & \multicolumn{2}{c}{ CS-Net } \\
\cline { 2 - 5 } & FDR & SE & FDR & SE \\
\hline Raw & $0.394 \pm 0.007$ & $0.738 \pm 0.010$ & $0.252 \pm 0.004$ & $0.842 \pm 0.003$ \\
\hline SSR & $0.398 \pm 0.015$ & $0.720 \pm 0.012$ & $0.251 \pm 0.008$ & $0.840 \pm 0.004$ \\
MSR & $0.375 \pm 0.012$ & $0.739 \pm 0.010$ & $0.256 \pm 0.007$ & $0.847 \pm 0.006$ \\
CLAHE & $0.375 \pm 0.009$ & $0.739 \pm 0.011$ & $0.249 \pm 0.006$ & $0.839 \pm 0.005$ \\
GIF & $0.372 \pm 0.007$ & $0.745 \pm 0.006$ & $0.246 \pm 0.011$ & $0.845 \pm 0.003$ \\
WVM & $0.370 \pm 0.009$ & $0.751 \pm 0.007$ & $0.246 \pm 0.005$ & $0.849 \pm 0.004$ \\
\hline Proposed & $\mathbf{0 . 3 6 1} \pm \mathbf{0 . 0 1 1}$ & $\mathbf{0 . 7 5 4} \pm \mathbf{0 . 0 0 4}$ & $\mathbf{0 . 2 4 0} \pm \mathbf{0 . 0 0 1}$ & $\mathbf{0 . 8 5 7} \pm \mathbf{0 . 0 0 1}$ \\
\hline
\end{tabular}

the CS-Net on randomly sampled $80 \%$ images from CCM-A, leaving out $20 \%$ of this dataset as a testing set.

In addition, we investigate how the proposed illumination correction method will affect conventional segmentation methods. The Infinite Perimeter Active Contour with Hybrid Region (IPACHR) method [49] was used for its effectiveness in detecting curvilinear objects (e.g., vessels and nerve fibers) with irregular and oscillatory boundaries. Any small and/or isolated objects were eliminated by the use of a disk-shaped opening operation with a radius of 2 pixels. For fair comparison the segmentation performance of IPACHR was evaluated on the test set used by the CS-Net.

To compare the segmentation performance of the proposed method with the corresponding ground truth, we compute the sensitivity (SE) and false discovery rate (FDR) between the predicted centerlines and ground truth ones. SE is the fraction of the number of pixels on the true positive nerves over the total number of pixels on the ground truth ones. FDR is defined as the fraction of the total number of pixels on the false positive nerves over the total number of pixels on the manually traced ones. The use of specificity, defined as the number of correctly classified pixels on the true negative class, is not adequate for the evaluation of this segmentation task, since the vast majority of pixels do not belong to corneal nerves. We should note that since the evaluation methods in general extract only one pixel-wide curves, a three-pixel tolerance region around the manually-traced nerves is considered to be true positive: in other words, a predicted centerline point is considered as true positive if it has at most three-pixels distant from a ground truth point.

The bottom row of Fig. 7 demonstrates the enhancementguided fiber segmentation results obtained by the IPACHR method. The benefit of the proposed enhancement method for segmentation may be observed from the representative region (yellow arrow). It may be seen that more completed fibers have been identified by our method. It achieves relatively uniform responses in both high- and low- intensity regions of the original image, and provides relatively more sensitive segmentation on small fibers than the other methods. The proposed enhancement method is not only able to correct intensity inhomogeneities, making fibers stand out more conspicuously from background, but also has the ability to reject nonfiber features. This is because the proposed method estimates reflectance and illumination simultaneously, allowing the noise term in Eqn. (5) to handle noise more effectively.

This finding is also evidenced by the segmentation performance illustrated in TABLE III. The proposed method improves the segmentation of the original images (raw) in CCM-A: by an increase of about 0.032 and 0.015 in SE, and a reduction of about 0.017 and 0.012 in FDR by the IPACHR and CS-Net segmentation methods, respectively. By contrast, relatively more significant margins of tracing results have been shown when the proposed method was compared with other enhancement methods, which indicate that our enhancement method has larger improvement than the other competitors. Because the proposed enhancement method is able to enhance the contrast between the nerve fibers and background, it reduces false detection more effectively, which in the end raises the sensitivity score. A statistical $t$-test indicates that the improvement of fiber tracing is significant with $p<0.001$ for both the IPACHR and CS-Net methods.

\section{B. Evaluation of Tortuosity Grading}

In this section, we validate the proposed tortuosity estimation method over two corneal nerve datasets.

1) Evaluation metrics: As suggested in [3], weighted sensitivity (wSe), specificity (wSp), and accuracy (wAcc) are employed to compare the classification results of different methods with ground truth. These metrics are defined as:

$$
\begin{gathered}
\mathrm{wSp}=\sum_{i=1}^{N} r_{i} \frac{\mathrm{TN}_{i}}{\mathrm{TN}_{i}+\mathrm{FP}_{i}}, \quad \mathrm{wSe}=\sum_{i=1}^{N} r_{i} \frac{\mathrm{TP}_{i}}{\mathrm{TP}_{i}+\mathrm{FN}_{i}}, \\
\mathrm{wAcc}=\sum_{i=1}^{N} r_{i} \frac{\mathrm{TP}_{i}+\mathrm{TN}_{i}}{\mathrm{TP}_{i}+\mathrm{TN}_{i}+\mathrm{FP}_{i}+\mathrm{FN}_{i}}
\end{gathered}
$$

where $i$ is the level of tortuosity, $\mathrm{TP}_{i}, \mathrm{TN}_{i}, \mathrm{FP}_{i}$, and $\mathrm{FN}_{i}$ indicate the true positives, true negatives, false positives, and false negatives, respectively over all the images available. $N$ denotes the number of levels/classes, and $r_{i}$ is the percentage of all the available images belonging to class/level $i$.

2) Tortuosity level classification: Discriminating between four tortuosity levels in CCM-A and three levels in CCM-B are multi-class classification problems. In order to demonstrate the superiority of the proposed tortuosity classification method, we computed the performance measure on a per-level and overall basis: the results are reported in TABLE IV. One state-of-theart tortuosity classification method [3] (referred to as M1 in TABLE IV) was re-implemented for the purpose of a comparative study: Annunziata et al. [3] included the DM, TDD, SCC, $\kappa_{a}$, and $\kappa_{a c}$ (see TABLE I) in their dictionary of features, and employed a weighted average operation to combine the image level features, and finally employed the Multinomial Logistic Ordinal Regression (MLOR) to classify the tortuosity level of 
TABLE IV

RESULTS OF PER-LEVEL TORTUOSITY CLASSIFICATION BY DIFFERENT APPROACHES. M1: ANNUNZIATA'S APPROACH [3]; M2: THE PROPOSED METHOD, WITHOUT THE ENHANCEMENT STEP; M3: THE PROPOSED METHOD; M4: THE PROPOSED METHOD APPLIED TO MANUAL FIBER SEGMENTATIONS.

\begin{tabular}{cl|ccccc|cccc}
\hline \hline & & \multicolumn{4}{|c|}{ CCM-A } & \multicolumn{4}{c}{ CCM-B } \\
\cline { 3 - 10 } & & level 1 & level 2 & level 3 & level 4 & overall & low & mid & high & overall \\
\hline \multirow{2}{*}{ M1 } & wSe & 0.718 & 0.644 & 0.660 & 0.707 & 0.663 & 0.783 & 0.743 & 0.761 & 0.766 \\
& wSp & 0.867 & 0.790 & 0.783 & 0.857 & 0.806 & 0.904 & 0.860 & 0.864 & 0.912 \\
& wAcc & 0.858 & 0.780 & 0.759 & 0.843 & 0.790 & 0.877 & 0.826 & 0.847 & 0.848 \\
\hline \multirow{2}{*}{$\mathbf{M} 2$} & wSe & 0.729 & 0.652 & 0.667 & 0.711 & 0.674 & 0.809 & 0.755 & 0.781 & 0.782 \\
& wSp & 0.876 & 0.801 & 0.793 & 0.864 & 0.813 & 0.913 & 0.863 & 0.884 & 0.920 \\
& wAcc & 0.864 & 0.786 & 0.771 & 0.851 & 0.796 & 0.908 & 0.831 & 0.869 & 0.869 \\
\hline \multirow{2}{*}{ M3 } & wSe & 0.740 & 0.673 & 0.681 & 0.732 & 0.711 & 0.821 & 0.761 & 0.787 & 0.801 \\
& wSp & 0.896 & 0.817 & 0.807 & 0.879 & 0.850 & 0.928 & 0.870 & 0.889 & 0.927 \\
& wAcc & 0.879 & 0.796 & 0.790 & 0.860 & 0.818 & 0.911 & 0.839 & 0.879 & 0.875 \\
\hline \multirow{2}{*}{ M4 } & wSe & 0.743 & 0.680 & 0.688 & 0.738 & 0.717 & 0.826 & 0.769 & 0.800 & 0.810 \\
& wSp & 0.901 & 0.801 & 0.814 & 0.881 & 0.859 & 0.932 & 0.876 & 0.894 & 0.932 \\
& wAcc & 0.884 & 0.803 & 0.800 & 0.866 & 0.824 & 0.918 & 0.843 & 0.882 & 0.879 \\
\hline \hline
\end{tabular}

each CCM image. Note, all the suggested parameters in [3] were used in our experiments.

TABLE IV also shows a comparison between the proposed method (referred to as M3) and Annunziata's approach [3] (referred to as M1) in terms of all performance measures on the enhanced images of CCM-A and CCM-B. Our method outperforms Annunziata's approach, with higher wSe, wSp, and wAcc scores. The proposed method achieved overall accuracies of 0.818 and 0.875 in CCM-A and CCM-B, respectively, while Annunziata's approach yielded lower performance, with overall accuracies of 0.790 and 0.848 , respectively. It is worth noting that careful observation shows that some tortuosity levels, such as grades 1 and 4 of CCM-A and the lower levels of CCM-B, may be easier to identify than the others, whether in terms of manually-segmented fibers (M4) or automated segmentation (M1-M3). High accuracies of 0.879, 0.860, and 0.911 were achieved by the proposed method, while 0.858 , 0.843 and 0.877 were obtained by Annunziata's approach. In our experiments, only 2 images from the lower level of CCM-B were incorrectly classified. A close inspection of these two images revealed that some nerve fibers were incompletely traced due to the pathology characteristics. By contrast, performances decreased for the medium levels, such as grades 2 and 3 in CCM-A, due to the smaller differences between them.

3) The effectiveness of image enhancement for tortuosity analysis: Fig. 9 demonstrates how the tortuosity analysis benefits from the proposed enhancement method using exponential curvature. Different from conventional curvature measurement which was performed on pre-segmented structures, the exponential curvature estimation produces a curvature map for all the pixels in an image, where nerve fiber structures have relatively high curvature values, while background pixels have curvature values close to zero. In Fig. 9, we can observe that the generated map also suffers from curvature values in the curvilinear shape of the artifacts in the background. However, after performing curvature estimation on the enhanced nerve fibers, we obtain a more accurate curvature map with more clearly visible fine structures and cleaner background, as shown in the bottom-right of Fig. 9.

We further validate the effectiveness of the proposed enhancement method for tortuosity analysis. TABLE IV reports
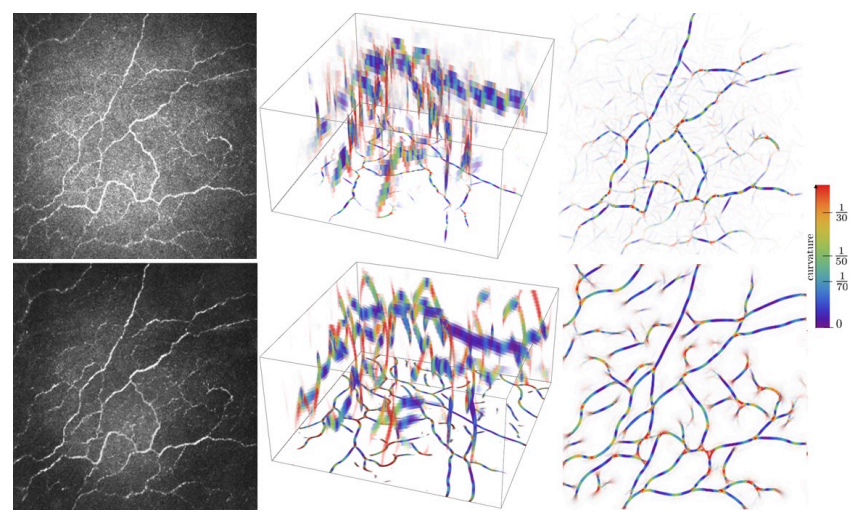

Fig. 9. The effect of the proposed enhancement method on the exponential curvature estimation. Left: original and enhanced images. Middle: Exponential curvature estimation. Right: Projected curvature.

the evaluation results in terms of the proposed method first without and then with the proposed enhancement method applied: these methods are referred to as M2 and M3, respectively. As can be observed, the proposed enhancement method assisted the tortuosity analysis in yielding higher performance in terms of all the metrics by significant margins, when compared with the direct application of the tortuosity analysis to the original images. The noise-constrained Retinex model contributed significantly to the final results, with an overall improvement of about 0.037 and 0.019 in wSe over CCM-A and CCM-B, respectively. In conclusion, the proposed noise-constrained Retinex model is helpful in improving the accuracy of tortuosity grading, since the correction of intensity inhomogeneities enhances the visibility of the fiber structures for subsequent processing.

4) The effectiveness of manual fiber segmentation for tortuosity analysis: As aforementioned, both sets of manually-traced fiber centerlines from datasets CCM-A and CCM-B are available from our clinical author. In order to characterize how the errors of both original images and manual fiber segmentations affect the results of tortuosity analysis, we report the tortuosity classification performances on manual segmentation (referred to as M4 in TABLE IV). These results show that our tortuosity estimation method is relatively stable whether applied to original images or to manual fiber seg- 
TABLE V

THE PROPOSED TUOTORSITY MEASUREMENT $\kappa_{\text {EXP IN }}$ CONTROL SUBJECTS AND OTHER DRY EYE DISEASE AND DIABETES PATIENTS.

\begin{tabular}{c|ccc}
\hline \hline Conditions & No. of patients & No. of images & $\kappa_{\exp }$ \\
\hline Healthy & 28 & 123 & 0.1706 \\
Dry eye & 28 & 124 & 0.2104 \\
Diabetes & 24 & 120 & 0.2396 \\
Dry eye and diabetes & 23 & 36 & 0.2453 \\
\hline
\end{tabular}

TABLE VI

SPEARMAN CORRELATION SCORES OF THE RETINAL BLOOD VESSEL TORTUOSITY MEASURES WITH AUTOMATIC AND MANUAL VESSEL SEGMENTATIONS.

\begin{tabular}{c|c|c|c|c|c}
\hline \hline \multirow{2}{*}{ Measures } & \multicolumn{2}{|c|}{ Manual segmentation } & \multicolumn{2}{|c}{ Automated segmentation } & \multirow{2}{*}{ Diff $^{\dagger}$} \\
\cline { 2 - 5 } & Arteries & Veins & Arteries & Veins & \\
\hline$L_{\chi}$ & 0.801 & 0.662 & 0.756 & 0.638 & 0.035 \\
$L_{c}$ & 0.813 & 0.701 & 0.784 & 0.677 & 0.027 \\
$\tau$ & 0.792 & 0.656 & 0.812 & 0.629 & 0.035 \\
$\kappa_{a}$ & 0.922 & 0.837 & 0.893 & 0.801 & 0.033 \\
$\kappa_{s}$ & 0.925 & 0.826 & 0.901 & 0.812 & 0.019 \\
$\kappa_{a c}$ & 0.919 & 0.814 & 0.877 & 0.768 & 0.044 \\
TD & 0.890 & 0.760 & 0.912 & 0.753 & 0.075 \\
TC & $\mathbf{0 . 9 4 9}$ & 0.853 & 0.919 & 0.812 & 0.036 \\
SCC & 0.850 & 0.770 & 0.827 & 0.745 & 0.024 \\
DCI & 0.787 & 0.589 & 0.734 & 0.621 & 0.101 \\
MAC & 0.820 & 0.814 & 0.801 & 0.795 & 0.019 \\
AAC & 0.838 & 0.695 & 0.841 & 0.677 & 0.075 \\
ICM & 0.684 & 0.575 & 0.661 & 0.542 & 0.028 \\
$\kappa_{\text {exp }}$ & 0.945 & $\mathbf{0 . 8 6 8}$ & $\mathbf{0 . 9 2 8}$ & $\mathbf{0 . 8 5 7}$ & $\mathbf{0 . 0 1 4}$ \\
\hline$\dagger$ & \multicolumn{7}{|c|}{ Diff $=$} & ave $\left(\mathrm{SC}_{\text {manual }}\right)-$ ave $\left(\mathrm{SC}_{\text {automated }}\right) \|$. &
\end{tabular}

mentations, where small differences of only 0.006 and 0.004 in overall accuracies were recorded over CCM-A and CCM-B, respectively.

5) Clinical evaluation: In order to demonstrate the capability in differentiating the health and pathology states, the proposed tortuosity measurement was further validated by the clinical practice. CCM-A dataset was used for evaluation, and it includes four groups based on the pathology conditions: 28 healthy subjects, 24 patients with diabetes, 28 subjects with dry eye disease, and 23 patients with both dry eye and diabetes. The clinical details of the study subjects and the proposed tortuosity measures are shown in TABLE V.

The results have demonstrated that the mean exponential curvature score of the healthy group is lower than those of all the other groups $(p<0.01)$, while that of the patients with both dry eye disease and diabetes is higher than those of all the other groups. These results indicate that the tuotorsity of nerve fibres measured by our exponential curvature has a potential to help in distinguishing the healthy subjects from those with diabetes or dry eye disease.

\section{Validation of Tortuosity Measurement}

In order to demonstrate the proposed tortuosity measure $\left(\kappa_{\exp }\right)$ is superior to other conventionally used measures, we further make a comparative study on a retinal vessel dataset: RET-TORT [8]. This dataset consists of images of 30 arteries and 30 veins of similar length and calibre, extracted from 60 retinal color fundus images taken from normal and hypertensive patients by the University of Padova, Italy. The acquired images were captured with a $50^{\circ}$ fundus camera (TRC 50, Topcon, Japan) with a resolution of $1100 \times 1300$ pixels. The images were manually ranked by a retinal specialist based on the degree of the vessel tortuosity.

Rather than relying on the tortuosity grading of the corneal nerves, the RET-TORT provides separate lists of images ordered by increasing tortuosity of arteries and veins, respectively. Since we are considering correlations among rankings, the Spearman Correlation was computed as a measure of fitness between automated and manual tortuosity grading. In addition, the automated grading results were further compared with those of the aforementioned tortuosity measures (see TABLE I). The result in TABLE VI shows that the proposed tortuosity measure has the highest correlation with automated grading in the assessment of both artery and vein tortuosities. It is worth noting that all the tortuosity measures require prior vessel segmentation, and the IPACHR model was employed again to generate the automated segmentation.

As we noted above, grading errors can propagate and accumulate due to image quality. Therefore, the SC scores of all the tortuosity measures over the manually-segmented retinal vessels are also reported in TABLE VI. This is a potential concern, because the IPACHR model utilizes a local phase operation, which may smooth the boundaries of the tortuous structures. This reservation was confirmed by the differences reported in the last column of TABLE VI. The SC scores of the conventional measures reveal significant differences between those performed on manual segmentations and those on automated segmentations. However, the SC scores of the proposed measure are only slightly lower than those on manual segmentation, resulting in a small difference of only 0.014. In summary, the above findings provide further evidence that the proposed tortuosity measure is less sensitive to curvilinear structure extraction methods than all the other measures studied.

\section{Discussion AND CONCLUSION}

It is worth noticing that inter-observer variability (IOV) is ubiquitous at many aspects of medical image analysis, such as the manual contour delineation of structures in CT [56], and fibre tortuosity estimation in the proposed task. With the rapid boost of deep learning-based medical imaging analysis, the IOV affects the development of deep learning-based methods from the training of network models to the evaluation of their performance [57]. As indicated in [56], the IOV in manual delineations for different structures in CT images is large and two or three observers may not be sufficient to establish the full range of IOV. Therefore, the conventional consensus amongst multiple observers is adopted in this paper.

Recently, a number of researchers focus on handling the uncertainty in deep learning-based segmentation, classification and registration. Several of them propose measurements which quantify uncertainties of deep learning methods [58]. In addition to the predictive uncertainty, the IOV is another major source of uncertainty in the supervised learning-based methods. Such uncertainty is typically reflected in the inconsistent annotations/labels of multiple observers and is independent of learning models and training algorithms. A common practice in the literature is to collect multiple annotations per sample 
and produce determined training samples with label fusion such as majority voting. This approach is particularly useful when inter-rater agreement is expected to be low [59]. A recent work [59] exploits IOV, where the uncertainty brought by IOV is treated as a target in supervised learning problem [58]. We conclude that since the evaluation of curvilinear structure segmentation is pixel-sensitive, it can be expected that the standardization of annotated positions on targeted curvilinear structures can be very helpful to increase the pixellevel agreements of multiple observers, and hence reduce the aleatoric uncertainty in IOV. This can be fulfilled by clarifying requirements of annotations to observers in protocol and automated standardizations of annotation results via data cleansing and augmentation.

Automated grading of the tortuosity level of nerve fibers or blood vessels in medical images still faces two major challenges. On one hand, there is no universally-accepted standard measure of tortuosity. On the other hand, traditional automated tortuosity grading is highly dependent on the quality of preprocessing: errors can be propagated and accumulated due to poor imaging quality and inaccurate results from the preprocessing pipeline.

To this end, we firstly proposed a new noise-constrained Retinex model to enhance CCM images, so as to address speckle noise, illumination inhomogeneities, and low contrast simultaneously. We modeled the noise term explicitly, and built and optimized an objective function for the estimation of the illumination, reflectance and noise components. We then adapted the orientation scores to lift and separate those crossed curvilinear structures in the enhanced image, defined a new tortuosity measure for these curvilinear structures and finally estimated their curvatures from their best fit curvilinear shape exponential curves in the orientation score space.

Our experimental results over three datasets demonstrate that the proposed tortuosity measure has performed better than conventional ones and that it has performed as well as a human expert. In addition, we have obtained manual annotations of nerve fiber tracing on two CCM datasets and constructed a new corneal nerve tortuosity dataset. All these datasets have been released for public access. As future work, we intend to investigate the importance of different features and employ other methods for the classification of the tortuosity of the corneal nerve fibers.

\section{REFERENCES}

[1] K. Edwards, N. Pritchard, D. Vagenas, A. Russell, R. A. Malik, and N. Efron, "Standardizing corneal nerve fibre length for nerve tortuosity increases its association with measures of diabetic neuropathy." Diabet. Med., vol. 31, no. 10, pp. 1205-9, 2014.

[2] J. Kim and M. Markoulli, "Automatic analysis of corneal nerves imaged using in vivo confocal microscopy." Clinical experimental optometry, vol. 101, no. 2, pp. 147-161, 2018.

[3] R. Annunziata, A. Kheirkhah, S. Aggarwal, P. Hamrah, and E. Trucco, "A fully automated tortuosity quantification system with application to corneal nerve fibres in confocal microscopy images," Med. Image Anal., vol. 32, pp. 216-232, 2016.

[4] Y. Zhao et al., "Retinal artery and vein classification via dominant sets clustering-based vascular topology estimation," in Proc. MICCAI, 2018, pp. 56-64.
[5] B. Williams et al., "An artificial intelligence-based deep learning algorithm for the diagnosis of diabetic neuropathy using corneal confocal microscopy: a development and validation study," Diabetologia, vol. 63, no. 2, pp. 419-430, 2020.

[6] P. Su et al., "Exploiting reliability-guided aggregation for the assessment of curvilinear structure tortuosity," in Proc. MICCAI, 2019, pp. 721-730.

[7] D. Vagenas et al., "Optimal image sample size for corneal nerve morphometry." Optometry and vision science, vol. 89, no. 5, pp. 812-7, 2012.

[8] E. Grisan, M. Foracchia, and A. Ruggeri, "A novel method for the automatic grading of retinal vessel tortuosity," IEEE Trans. Med. Imaging, vol. 27, pp. 310-319, 2008.

[9] F. Scarpa, X. Zheng, Y. Ohashi, and A. Ruggeri, "Automatic evaluation of corneal nerve tortuosity in images from in vivo confocal microscopy." Invest. Ophthal. Vis. Sci., vol. 52, no. 9, pp. 6404-8, 2011.

[10] N. Pritchard, K. Edwards, A. W. Russell, B. A. Perkins, R. A. Malik, and N. Efron, "Corneal confocal microscopy predicts 4-year incident peripheral neuropathy in type 1 diabetes." Diabetes care, vol. 38 , no. 4 , pp. 671-5, 2015.

[11] Y. Zhao et al., "Intensity and compactness enabled saliency estimation for leakage detection in diabetic and malarial retinopathy," IEEE Trans. Med. Imaging, vol. 36, no. 1, pp. 51-63, 2016.

[12] — "Retinal vascular network topology reconstruction and artery/vein classification via dominant set clustering," IEEE Trans. Med. Imaging, vol. 39, no. 2, pp. 341-356, 2020.

[13] R. Annunziata, A. Kheirkhah, S. Aggarwal, B. Cavalcanti, P. Hamrah, and E. Trucco, "Tortuosity classification of corneal nerves images using a multiple-scale-multiple-window approach," in Proc. MICCAI workshop OMIA, 2014, pp. 113-120.

[14] Y. Zhao et al., "Uniqueness-driven saliency analysis for automated abnormalities detection with application to retinal diseases," in Proc. MICCAI, 2018, pp. 109-118.

[15] J. Xie et al., "Topology reconstruction of tree-like structure in images via structural similarity measure and dominant set clustering," in Proc. CVPR, 2019, pp. 8505-8513.

[16] E. Bullitt, G. Gerig, S. M. Pizer, W. Lin, and S. R. Aylward, "Measuring tortuosity of the intracerebral vasculature from mra images," IEEE Trans. Med. Imaging, vol. 22, pp. 1163-1171, 2003.

[17] F. Scarpa and A. Ruggeri, "Development of clinically based corneal nerves tortuosity indexes," in Proc. MICCAI workshop OMIA, 2017, pp. 219-226.

[18] D. Bracher, "Changes in peripapillary tortuosity of the central retinal arteries in newborns," Graefe's Archive for Clinical and Experimental Ophthalmology, vol. 218, pp. 211-217, 1982.

[19] O. Smedby, N. HÃúgman, S. Nilsson, U. Erikson, A. Olsson, and G. Walldius, "Two-dimensional tortuosity of the superficial femoral artery in early atherosclerosis." Journal of vascular research, vol. 30 4, pp. 181-91, 1993.

[20] K. Goh, W. Hsu, M. Lee, and H. Wang, "Adris: an automatic diabetic retinal image screening system," Studies in Fuzziness and Soft Computing, vol. 60, pp. 181-210, 2001.

[21] E. Bribiesca, "A measure of tortuosity based on chain coding," Patter. Recogn., vol. 46, no. 3, pp. 716-724, 2013.

[22] W. E. Hart et al., "Measurement and classification of retinal vascular tortuosity," Int. Jour. Med. Infor., vol. 53, no. 2-3, pp. 239-52, 1999.

[23] Y. Zhao et al., "Automatic 2D/3D vessel enhancement in multiple modality images using a weighted symmetry filter," IEEE Trans. Med. Imaging, vol. 37, no. 2, pp. 438-450, 2018.

[24] E. H. Land and J. J. McCann, "Lightness and retinex theory." Journal of the Optical Society of America, vol. 61, no. 1, pp. 1-11, 1971.

[25] M. Elad, "Retinex by two bilateral filters," in Scale-Space, 2005.

[26] Y. Park and J. Kim, "Retinex method based on adaptive smoothing for illumination invariant face recognition," Signal Processing, vol. 88, no. 8, pp. 1929-1945, 2008.

[27] Y. Zhao et al., "Region-based saliency estimation for $3 \mathrm{~d}$ shape analysis and understanding," Neurocomputing, vol. 197, pp. 1-13, 2016.

[28] Y. Zhao, Y. Liu, R. Song, and M. Zhang, "A retinex theory based points sampling method for mesh simplification," in Proc. ISPA, 2011, pp. $230-235$.

[29] S. Setty, N. K. Srinath, and M. C. Hanumantharaju, "Development of multiscale retinex algorithm for medical image enhancement based on multi-rate sampling," in Proc. Int. Conf. Signal Process. Image Process. Pattern Recog., 2013, pp. 145-150.

[30] F. Tao, X. Yang, W. Wu, K. Liu, Z. Zhou, and Y. Liu, "Retinex-based image enhancement framework by using region covariance filter,' Soft Comput., vol. 22, no. 5, pp. 1399-1420, 2018. 
[31] X. Fu, Y. Liao, D. Zeng, Y. Huang, X. Zhang, and X. Ding, "A probabilistic method for image enhancement with simultaneous illumination and reflectance estimation," IEEE Trans. Image Process., vol. 24, pp. 4965-4977, 2015.

[32] M. Li, J. Liu, W. Yang, X. Sun, and Z. Guo, "Structure-revealing lowlight image enhancement via robust retinex model," IEEE Trans. Image Process., vol. 27, pp. 2828-2841, 2018.

[33] T. Goldstein and S. Osherg, "The split bregman method for 11regularized problems," SIAM J. Imaging Sci., vol. 2, no. 2, pp. 323-343, 2009.

[34] Y. Wang, W. Yin, and J. Zeng, "Global convergence of admm in nonconvex nonsmooth optimization," UCLA CAM Report, vol. 15, no. 62,2015

[35] Z. Rao, T. Xu, J. Luo, J. Guo, G. Shi, and H. Wang, "Non-uniform illumination endoscopic imaging enhancement via anti-degraded model and 1112-based variational retinex," EURASIP Journal on Wireless Communications and Networking, vol. 2017, pp. 1-11, 2017.

[36] R. Kimmel, M. Elad, D. Shaked, R. Keshet, and I. Sobel, "A variational framework for retinex," International Journal of Computer Vision, vol. 52, pp. 7-23, 2003.

[37] Y. Zhao, Y. Liu, X. Wu, S. P. Harding, and Y. Zheng, "Retinal vessel segmentation: An efficient graph cut approach with retinex and local phase," PLoS One, vol. 10, no. 4, p. 0122332, 2015.

[38] E. Franken and R. Duits, "Crossing-preserving coherence-enhancing diffusion on invertible orientation scores," Int. J. Comput. Vis., vol. 85 , no. 3, pp. 253-278, 2009.

[39] E. Bekkers, R. Duits, T. Berendschot, and B. Ter Haar Romeny, "A multi-orientation analysis approach to retinal vessel tracking," J. Math. Imaging Vis., vol. 49, no. 3, pp. 583-610, 2014.

[40] E. J. Bekkers, J. Zhang, R. Duits, and B. M. Haar, "Curvature based biomarkers for diabetic retinopathy via exponential curve fits in $\operatorname{SE}(2)$," in Proc. MICCAI, 2015, pp. 113-120.

[41] J. Zhang, B. Dashtbozorg, E. Bekkers, J. Pluim, R. Duits, and T. H. R. B., "Robust retinal vessel segmentation via locally adaptive derivative frames in orientation scores," IEEE Trans. Med. Imaging, vol. 35, pp. 2631-44, 2016

[42] C. Heneghan, J. Flynn, M. O'Keefe, and M. Cahill, "Characterization of changes in blood vessel width and tortuosity in retinopathy of prematurity using image analysis," Med. Image Anal., vol. 6, no. 4, pp. 407-29, 2002.

[43] T. Holmes et al., "Automated software analysis of corneal micrographs for peripheral neuropathy." Invest. Ophthal. Vis. Sci., vol. 51, no. 9, pp. 4480-91, 2010.

[44] P. Kallinikos, M. Berhanu, C. O'Donnell, A. Boulton, N. Efron, and R. Malik, "Corneal nerve tortuosity in diabetic patients with neuropathy." Invest. Ophthal. Vis. Sci., vol. 45, no. 2, pp. 418-22, 2004.

[45] E. Bribiesca and G. Bribiescacontreras, "2d tree object representation via the slope chain code," Patter. Recogn., vol. 47, no. 10, pp. 3242-3253, 2014.

[46] M. Patasius et al., "Evaluation of tortuosity of eye blood vessels using the integral of square of derivative of curvature," in Proc. EMBC, 2005.

[47] K. Chandrinos, M. Pilu, R. Fisher, and P. Trahanias, "Image processing techniques for the quantication of atherosclerotic changes," in Proc. the 8th Mediterranean Conf. on Medical and Biological Engineering and Computing, 1998.

[48] L. Oliveira-Soto and N. Efron, "Morphology of corneal nerves using confocal microscopy." Cornea, vol. 20, no. 4, pp. 374-84, 2001.

[49] Y. Zhao, L. Rada, K. Chen, S. Harding, and Y. Zheng, "Automated vessel segmentation using infinite perimeter active contour model with hybrid region information with application to retinal images," IEEE Trans. Med. Imaging, vol. 34, pp. 1797-1807, 2015.

[50] D. Jobson, Z. Rahman, and G. Woodell, "Properties and performance of a center/surround retinex," IEEE Trans. Med. Imaging, vol. 6, no. 3 pp. 451-62, 1997 .

[51] T. Okuno and T. Nishitani, "Efficient multi-scale retinex algorithm using multi-rate image processing," in Proc. ICIP, 2009, pp. 3145-3148.

[52] M. Abdullah-Al-Wadud, M. Kabir, M. Dewan, and O. Chae, "A dynamic histogram equalization for image contrast enhancement," IEEE Transactions on Consumer Electronics, vol. 53, no. 2, pp. 593-600, 2007.

[53] K. He, J. Sun, and X. Tang, "Guided image filtering," IEEE Trans. Patt. Anal. Machin. Intelligen., vol. 35, pp. 1397-1409, 2013.

[54] X. Fu, D. Zeng, Y. Huang, X. P. Zhang, and X. Ding, "A weighted variational model for simultaneous reflectance and illumination estimation," in Proc. CVPR, 2016, pp. 2782-2790.

[55] L. Mou et al., "Cs-net: Channel and spatial attention network for curvilinear structure segmentation," in Proc. MICCAI, 2019, pp. 721730
[56] L. Joskowicz, D. Cohen, N. Caplan, and J. Sosna, "Inter-observer variability of manual contour delineation of structures in ct," European Radiology, vol. 29, no. 3, p. 1391âĂŞ1399, 2019.

[57] L. Maier-Hein et al., "Why rankings of biomedical image analysis competitions should be interpreted with care," Nat Commun., vol. 9, no. 1, p. $5217,2018$.

[58] M. Jensen, D. Jorgensen, R. Jalaboi, M. Hansen, and M. Olsen, "Improving uncertainty estimation in convolutional neural networks using inter-rater agreement," in Proc. MICCAI, 2019, pp. 540-548.

[59] S. Hu, D. Worrall, S. Knegt, B. Veeling, H. Huisman, and M. Welling, "Supervised uncertainty quantification for segmentation with multiple annotations," in Proc. MICCAI, 2019, pp. 137-145. 
$2020-02-17$

\section{Automated tortuosity analysis of nerve fibers in corneal confocal microscopy}

Zhao, Yitian

IEEE

Zhao Y, Zhang J, Pereira E, et al., (2020) Automated tortuosity analysis of nerve fibers in corneal confocal microscopy, IEEE Transactions on Medical Imaging, Volume 39, Issue 9, September 2020, pp. 2725-2737

https://doi.org/10.1109/TMI.2020.2974499

Downloaded from Cranfield Library Services E-Repository 